\title{
Short-and long-term results of ultrasound-guided fine- needle aspiration and steroid injection in the treatment of ganglion cysts
}

\author{
ㄷTaha Yusuf Kuzan'1, @Osman Yüksel Yavuz² \\ ${ }^{1}$ Sancaktepe Şehit Prof. Dr. İlhan Varank Training and Research Hospital, Department of Radiology, İstanbul, Turkey \\ ${ }^{2}$ Sancaktepe Şehit Prof. Dr. İlhan Varank Training and Research Hospital, Department of Orthopedics and Traumatology, İstanbul, Turkey
}

Cite this article as: Kuzan TY, Yavuz OY. Short-and long-term results of ultrasound-guided fine-needle aspiration and steroid injection in the treatment of ganglion cysts. J Health Sci Med 2022; 5(1): 269-273.

\begin{abstract}
Objective: To determine the efficacy and safety of percutaneous aspiration and steroid injection treatment for ganglion cysts and retrospectively evaluate the size change in ganglion cysts during follow-up.

Material and Method: All patients who underwent percutaneous aspiration and steroid injection treatment for ganglion cysts between January 2019 and February 2020 were included in the study. Demographic characteristics and clinical signs and findings of the patients were obtained from electronic patient records. Treatment response was classified as "excellent," "good," and "poor" at one month and one year according to ultrasound measurement.

Results: Ganglion cyst aspiration and steroid injection were successfully performed in 37 (86.0\%) of 43 cases. A total of 28 cases with short- and long-term follow-ups were included in the final analysis. All the cases had cosmetic complaints before the treatment, and seven had pain and four had limited range of motion. Early treatment response was excellent in 7 (25.0\%) patients, good in $14(50.0 \%)$, and poor in $7(50.0 \%)$. Late treatment response was excellent in 18 (64.3\%) patients, good in 3 $(10.7 \%)$, and poor in $7(25.0 \%)$. After the procedure, mild pain lasting less than two days developed in the cyst area in half of the patients. Skin color change was observed in two cases in the first-month follow-up.

Conclusion: Ultrasound-guided fine-needle aspiration and steroid injection with the right technique is an alternative costeffective method to surgery that can be successfully applied in the treatment of ganglion cysts with low side effects and relatively good results.
\end{abstract}

Keywords: Ganglion, cyst, aspiration, steroid, injection

\section{INTRODUCTION}

Ganglion cysts are benign "tumor-like" fluid lesions that develop because of mucinous involution of collagen structures; they may originate from different parts of the body in relation to the musculoskeletal system, such as joint capsules, muscles, tendons, and tendon sheaths $(1,2)$. They are most often seen on the dorsal side of the wrist (70\%), followed by the volar side of the wrist (20\%), and in the tendon sheath of the fingers, while ganglion cysts originating from other joints are relatively rare $(3,4)$. Most ganglion cysts are asymptomatic except for swelling. When symptomatic they result in pain, effusion, joint tenderness, and limited range of motion (2). Although ganglion cysts are non-malignant cystic masses, most patients seek treatment because of their cosmetic appearance or because of concerns about their ganglion enlargement.
Treatment options include non-invasive follow-up, conservative treatment, and surgical excision (5). Although there are publications on conservative care of ganglion cysts, there is scarce information in the literature on the results of treatment with ultrasoundguided percutaneous aspiration and steroid injection (6).

Our aim in this study is to determine the efficacy and safety of treatment in patients who underwent percutaneous aspiration and steroid injection due to ganglion cysts in our center, and to evaluate retrospectively the size change in ganglion cysts during the follow-up period. 


\section{MATERIAL AND METHOD}

The study was carried out with the permission Sancaktepe Sehit Prof. Dr. İlhan Varank Training and Research Hospital Ethics Committee (Date: 2021, Decision No: 2021/89). All procedures were carried out in accordance with the ethical rules and the principles of the Declaration of Helsinki.

\section{Patient Selection and Study Design}

All patients who underwent percutaneous treatment for ganglion cysts in the interventional radiology department of our center between January 2019 and February 2020 were included in this retrospective study. Demographic, clinical, and radiological characteristics and treatment and outcomes data were obtained from electronic medical records. The dominant hand of the patients was also noted. All patients included in the study underwent ultrasonography (USG) by a general radiologist for ganglion cysts. The ganglion cyst diagnosis on ultrasound was made by showing the connection of the anechoic-hypoechoic cyst with a typical localization, well-defined margins, and acoustic enhancement with the adjacent joint. In doubtful cases on USG, the diagnosis was confirmed by magnetic resonance imaging (MRI). The definitive diagnosis was confirmed by thick and clear or translucent gelatinous fluid aspirated from the cysts. Before the procedure, the volume of the cysts was calculated and recorded on USG (with the formula: volume = width $\times$ depth $\times$ length $\times 0.52$ ). The study did not include cysts with a long axis shorter than $1 \mathrm{~cm}$ and patients who could not be aspirated or did not receive a steroid injection after aspiration.

The patients were called for follow-up one month and one year after the procedure to evaluate their response to early and long-term treatments, respectively. USG was conducted again to allow for the measurement of volume and comparison with the previous USG. Treatment response was defined by USG volume measurement: 1: Excellent - complete regression without induration, 2: Good - > 50\% volume regression, 3: Poor $-<50 \%$ volume regression or size increase. The interventional radiologist made baseline and follow-up USG measurements.

Cases with short-term and long-term follow-ups were included in the final analysis. The flow chart of the study is shown in Figure 1.

\section{Ultrasound-guided Fine-needle Aspiration and Steroid Injection Technique}

Ganglion cyst aspiration and steroid injections were performed by a radiologist with seven years of experience in all cases. All procedures were performed under USG guidance using a linear 7.2-14 MHz probe. Before the procedure, the cyst was detected using the ultrasound probe, and a local anesthetic was applied to the skin. Having allowed the appropriate time for the topical anesthesia to be effective, the skin was then cleaned with iodine solution. Before the cyst was punctured, it was softened by applying light external pressure. Then, under USG guidance, the center of the cyst was entered using a $21 \mathrm{G}$ fine needle, and the cyst fluid was aspirated as much as possible with a $10 \mathrm{ml}$ disposable luer-lock injector. In cases where aspiration of the entire cyst was not possible, external pressure was applied and the entire contents of the cyst were cleaned out. After aspiration, an intralesional $1 \mathrm{ml}$ steroid (Diprospan, Betamethasone dipropionate: $6.43 \mathrm{mg}$ (5.0 $\mathrm{mg}$ betamethasone equivalent) + Betamethasone sodium phosphate: $2.63 \mathrm{mg}$ (5.0 mg betamethasone)) was injected into the cyst through the same needle. After the procedure, a pressure bandage was applied to the cyst area. If pain then occurred, oral paracetamol was recommended. An example of a percutaneous treatment procedure and images of a ganglion cyst are shown in Figures 2 and 3.

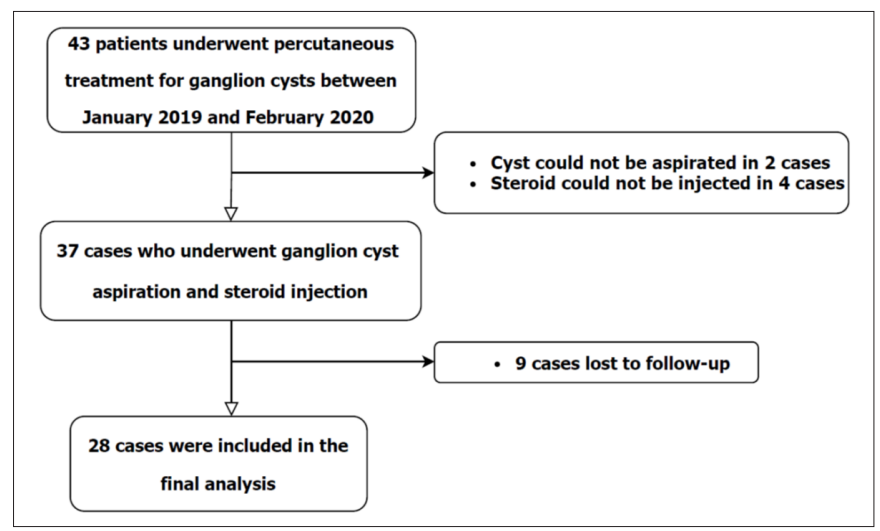

Figure 1. Flow chart of the study

\section{Statistical Analysis}

Descriptive analyzes were performed for patient characteristics. Normality control of continuous variables was done with Shapiro Wilk test. Normally distributed continuous variables were expressed as \pm standard deviation (SD), non-normally distributed continuous variables were expressed as median and range. Categorical variables were shown as percentages. Wilcoxon Signed-Rank test was used for cyst volume comparisons. P value $<0.05$ was considered statistically significant and analyzes were performed using SPSS statistics 23.0 software (IBM Corp., Armonk, NY, USA). 


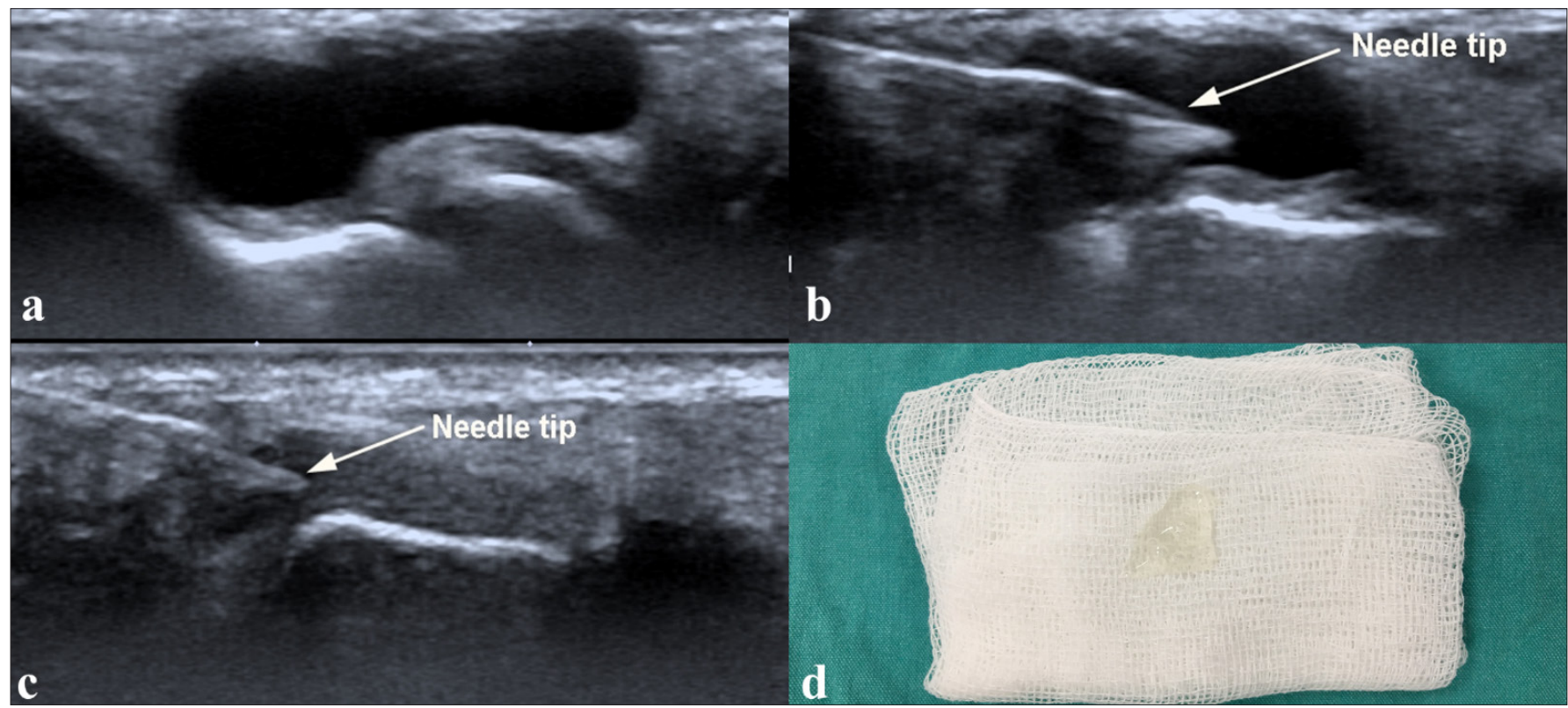

Figure 2. Ultrasonography image of the wrist ganglion cyst before the procedure (a). Under the guidance of ultrasonography, a 21 gauge needle is inserted into the central part of the ganglion cyst (b), the entire cyst content is aspirated (c), and then steroid injection is performed through the same needle tract. Clear and viscous translucent gelatinous fluid content of the ganglion cyst is seen (d).

\section{RESULTS}

A total of 28 cases with short- and long-term followups were included in the final analysis. The age of the cases ranged from 16 to 60 , with a median of 29.9 years. Ten cases $(35.7 \%)$ were male and the remaining $(64.3 \%)$ were female. The cysts were located in the dorsal of the right hand in 17 (60.7\%) cases, the left hand dorsal in $8(28.6 \%)$ cases, the left foot dorsal in $2(7.1 \%)$ cases, and the right foot dorsal in $1(3.6 \%)$ case. 7 of the cases had pain and 4 had limited range of motion while all of the cases had cosmetic complaints before the treatment. Of the cases with wrist ganglion, 20 were right hand dominant and 5 left hand dominant.

In 2 of the cases that were not included in the final analysis, the cyst content could not be aspirated completely as it was too viscous, apart from this, cyst aspiration was technically successful in 41 (95.3\%) of 43 patients. Steroid injection could not be performed after aspiration in one case because the needle tract was lost after aspiration and in 3 cases because the aspiration volume was too small. As a result, aspiration and steroid injection of ganglion cysts were technically successful in $37(86.0 \%)$ of 43 cases. Nine out of 37 patients were not included in the final analysis because they did not participate in the follow-ups.

Early and late treatment responses are summarized in Table 1 . The difference between the early $(383.3 \pm 631.1$ $\left.\mathrm{mm}^{3}\right)$ and late $\left(282.9 \pm 618.1 \mathrm{~mm}^{3}\right)$ volumes compared to the baseline (was $995.5 \pm 736.8 \mathrm{~mm}^{3}$ ) volume was quite significant $(\mathrm{p}<0.001$ and $\mathrm{p}<0.001$, respectively). However, the difference in volume between the early and late periods was not significant $(\mathrm{p}=0.530)$.

\begin{tabular}{|lcccc|}
\hline Table 1. First month and first-year treatment response status \\
\cline { 2 - 4 } & \multicolumn{3}{c|}{$\mathbf{1}^{\text {st }}$ year follow-up } & \\
\cline { 2 - 4 } & Excellent & Good & Poor & Total \\
\hline $1^{\text {st }}$ month follow-up & & & & \\
Excellent & 5 & 1 & 1 & 7 \\
Good & 9 & 1 & 4 & 14 \\
Poor & 4 & 1 & 2 & 7 \\
Total & 18 & 3 & 7 & 28 \\
\hline
\end{tabular}

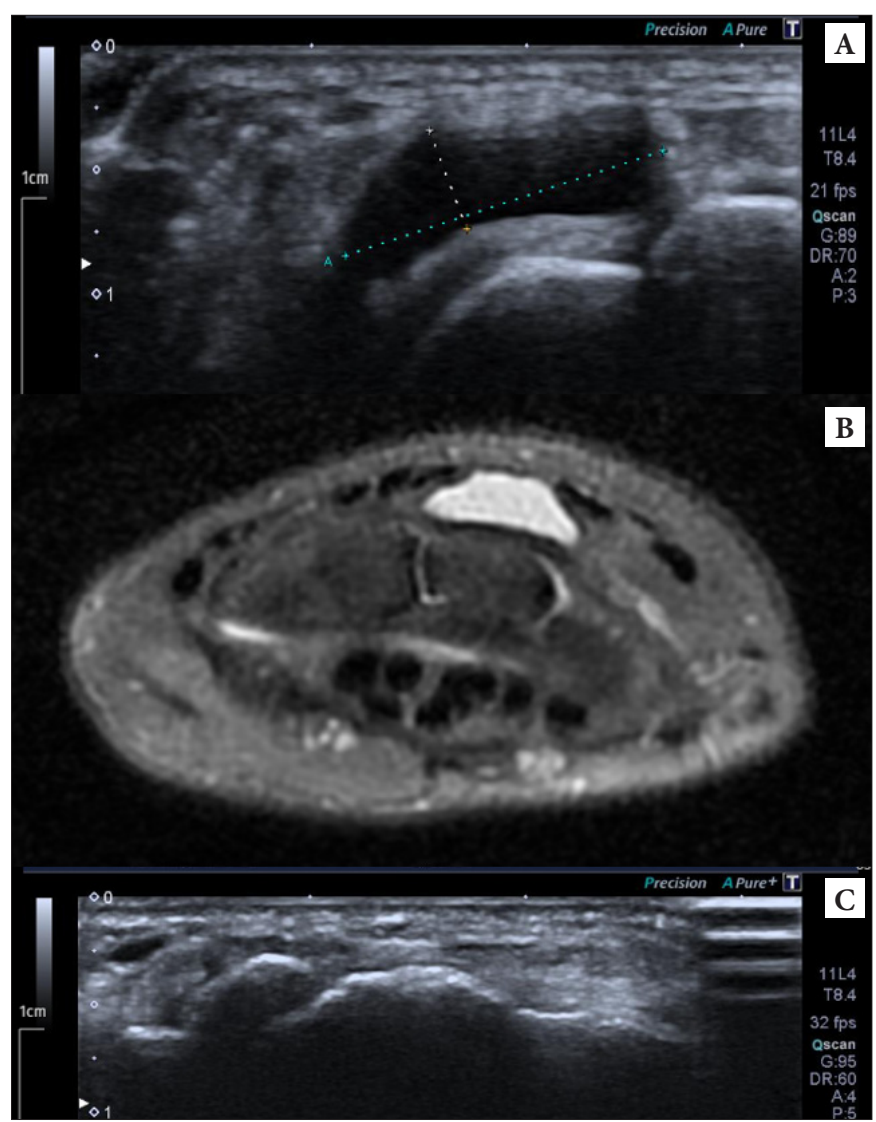

Figure 3. Ultrasonography (3a) and T2-weighted magnetic resonance imaging $(3 \mathrm{~b})$ of the patient with dorsal wrist ganglion. After the percutaneous treatment, the first-month follow-up ultrasonography (3c) showed that the cyst had completely disappeared. 
Mild pain lasting less than 2 days developed in the cyst area in half of the cases after the procedure. In 2 cases, skin color change was observed in the $1^{\text {st }}$-month followup. In the $1^{\text {st }}$ year follow-up, the color change improved in one of the cases.

\section{DISCUSSION}

In this study, the outcomes of percutaneous aspiration and steroid injection in the treatment of ganglion cysts were analyzed. Both the early and late responses of aspiration and steroid injection were found to be acceptable in three out of four cases.

The reason for the formation of ganglion cysts is not known precisely; one of the theories is that ganglion cysts are caused by chronic inflammation. Based on this assumption, steroid injection is preferred for the treatment of ganglion cysts (7). However, conflicting results have been reported on the effectiveness of this treatment method. In addition, there is no standard for the type, amount and method of administration of the steroid. The aim of this study was planned to clarify these points.

Similar to the literature, the patients included in the study were mostly women, and their lesions were located mostly in the dorsal wrist. What is remarkable in our study was that the patients' ganglion cysts were twice as likely to be located in the right hand as in the left hand. Turgut et al. similarly reported more ganglion cysts in the right hand and associated this with the dominant hand (8). In our study, most of the patients were right-hand dominant; thus, their ganglion cysts may be related to repetitive injury, which is one of the formation theories of ganglion cysts (9).

Some researchers have suggested that $40-58 \%$ of ganglion cysts can regress spontaneously, and therefore, patients who do not request intervention can be followed (10). However, as in our study, patients often want to be treated primarily for cosmetic reasons and less often because the ganglia are symptomatic. Although different surgical and non-surgical methods are used to treat ganglion cysts, there is no standard for the management of ganglion cysts. Treatment options include open or arthroscopic surgical excision, observation and percutaneous methods such as aspiration with or without steroid or hyaluronidase injections and sclerotherapy (4), but the treatment option that is best for ganglion cyst management remains uncertain.

The literature reports different results in the treatment of ganglion cysts with both different and similar methods. In a review evaluating the efficacy of percutaneous methods, it was shown that more than half of ganglion cysts recur after aspiration alone (4). Although Mackie et al. (11) showed that sclerosis of ganglion cysts with sodium tetradecyl disulphate resulted in recurrence in up to $94 \%$ of cases, other studies have found that sclerosis with newer methods has very low failure rates (12). While Becker et al. (13) claim that aspiration and steroid injection provide resolution as high as $87 \%$, Varley et al. (6) claim that steroid injection does not make any additional contribution to aspiration. In our study, we attributed the fact that the majority of patients who reported good results in the early period, showed excellent results in the late period to the long-term effect of steroid injection. In studies evaluating the results of percutaneous aspiration and steroid injection of ganglion cysts, the recurrence rate was found to be between 35\% and $83 \%$ (14). In our study, an acceptable ganglion cyst resolution of $75 \%$ was achieved in the first year, similar to the results of Becker et al. (13).

Some previous studies have found that the recurrence rate after open or arthroscopic surgery ranges from $0 \%$ to $31.2 \%(4,15,16)$. Although arthroscopic surgery is among the treatment options, in a wrist arthroscopy series that included 2,420 patients, an eight-fold increase in ganglion cyst development was reported after arthroscopy compared to the normal population (17). Recurrence of surgery was found to be lower in two randomized controlled studies that compared surgery with percutaneous treatment $(18,19)$. Although the recurrence of surgery shows a variable range, the general opinion is that the recurrence of surgery is less than aspiration, but the complication rates are significantly higher in surgery compared to aspiration $(0-56 \%$ versus $0-12 \%)(4,16,20)$.

In our study, short-term mild pain was observed in onefourth of the patients after the procedure. In 2 cases, skin depigmentation was observed. Skin depigmentation is one of the side effects of steroids and has been observed in similar studies using steroid injection (21). In this study, the patients who developed pain and depigmentation were the patients whose aspiration volume was less than the injected steroid. Therefore, the authors agree that side effects such as post-procedure pain and depigmentation are due to the injection of the steroid coming out of the cyst cavity. To reduce the side effects that develop due to the injected steroid; multiple punctures should not be performed on the cyst to be aspirated, the same inlet tract should be used for steroid injection, and most importantly, steroid injection should not be made more than the aspirated ganglion content. Because steroids that exceed the cyst capacity or spread out of the cyst cavity can cause pain, skin atrophy and depigmentation.

Although the treatment success of surgical excision is relatively high, the complications of the procedure are serious and excessive compared to percutaneous methods. 
In recurrent cysts, reoperation is not always possible. For this reason, ultrasound-guided percutaneous aspiration of ganglion cysts can be performed quickly, safely, and cheaper in outpatient settings (22). Another advantage of ultrasound-guided aspiration is that the procedure is reproducible.

However, some limitations should be noted. First, some patients avoided coming to the hospital and did not participate in the follow-up because the study time coincided with the pandemic period. This resulted in a relative decrease in the number of patients included in the final analysis. Second, MRI was not performed in all of the patients before the procedure, and the diagnosis was made only by USG in these patients. Another limitation is the relative shortness of the follow-up period.

\section{CONCLUSION}

As a result, ultrasound-guided fine-needle aspiration and steroid injection with the right technique; is an alternative cost-effective method to surgery that can be successfully applied in the treatment of ganglion cysts with low side effects and relatively good results.

\section{ETHICAL DECLARATIONS}

Ethics Committee Approval: The study was carried out with the permission Sancaktepe Şehit Prof. Dr. İlhan Varank Training and Research Hospital Ethics Committee (Date: 2021, Decision No: 2021/89).

Informed Consent: All patients signed the free and informed consent form.

Referee Evaluation Process: Externally peer-reviewed.

Conflict of Interest Statement: The authors have no conflicts of interest to declare.

Financial Disclosure: The authors declared that this study has received no financial support.

Author Contributions: All of the authors declare that they have all participated in the design, execution, and analysis of the paper, and that they have approved the final version.

\section{REFERENCES}

1. Jose J, Silverman E, Kaplan L. Symptomatic ganglion cyst of the popliteus tendon treated with ultrasound-guided aspiration and steroid injection: A case report. Sports Health 2011; 3: 393-5.

2. Derman P, Kamath AF, Kelly Iv JD. Ganglion cysts of the posterior cruciate ligament. Am J Orthop (Belle Mead NJ) 2011; 40: 257-8.

3. DeFriend DE, Schranz PJ, Silver DA. Ultrasound-guided aspiration of posterior cruciate ligament ganglion cysts. Skeletal Radiol 2001; 30: 411-4.

4. Suen M, Fung B, Lung CP. Treatment of ganglion cysts. ISRN Orthop 2013; 2013: 940615.
5. Gude W, Morelli V. Ganglion cysts of the wrist: pathophysiology, clinical picture, and management. Curr Rev Musculoskelet Med 2008; 1: 205-11.

6. Varley GW, Needoff M, Davis TR, Clay NR. Conservative management of wrist ganglia. Aspiration versus steroid infiltration. J Hand Surg Br 1997; 22: 636-7.

7. Breidahl WH, Adler RS. Ultrasound-guided injection of ganglia with coricosteroids. Skeletal Radiol 1996; 25: 635-8.

8. Turgut MC, Toy S. Surgical outcomes regarding wrist ganglion cysts based on surgical experience. Erciyes Med J 2021. doi: 10.14744/etd.2021.38739

9. Nahra ME, Bucchieri JS. Ganglion cysts and other tumor related conditions of the hand and wrist. Hand Clin 2004; 20: 249-60.

10.Dias JJ, Dhukaram V, Kumar P. The natural history of untreated dorsal wrist ganglia and patient reported outcome 6 years after intervention. J Hand Surg Eur Vol 2007; 32: 502-8.

11. Mackie IG, Howard CB, Wilkins P. The dangers of sclerotherapy in the treatment of ganglia. J Hand Surg Br 1984; 9: 181-4.

12. Gümüş N. A new sclerotherapy technique for the wrist ganglion: Transcutaneous electrocauterization. Ann Plast Surg 2009; 63: $42-4$.

13. Becker WF. Hydrocortisone therapy in ganglia. Ind Med Surg 1953; 22: 555-7.

14. Hussain S, Akhtar S, Aslam V, Khan SM. Efficacy of aspiration and steroid injection in treatment of ganglion cyst. Pakistan J Med Heal Sci 2015; 9: 1403-5.

15. Kim JP, Seo JB, Park HG, Park YH. Arthroscopic excision of dorsal wrist ganglion: Factors related to recurrence and postoperative residual pain. Arthrosc - J Arthrosc Relat Surg 2013; 29: 1019-24.

16. Gündeș H, Cirpici Y, Sarlak A, Müezzinoglu S. Prognosis of wrist ganglion operations. Acta Orthop Belg 2000; 66: 363-7.

17. Rochlin DH, Perrault D, Sheckter CC, Fox P, Yao J. Prevalence of Ganglion Cyst Formation After Wrist Arthroscopy: A Retrospective Longitudinal Analysis of 2420 Patients. Hand 2020.

18. Jagers Op Akkerhuis $M$, Van Der Heijden M, Brink PRG. Hyaluronidase versus surgical excision of ganglia: a prospective, randomized clinical trial. J Hand Surg Br 2002; 27: 256-8.

19.Limpaphayom N, Wilairatana V. Randomized controlled trial between surgery and aspiration combined with methylprednisolone acetate injection plus wrist immobilization in the treatment of dorsal carpal ganglion. J Med Assoc Thai 2004; 87: 1513-7.

20. Singhal R, Angmo N, Gupta S, Kumar V, Mehtani A. Ganglion cysts of the wrist: a prospective study of a simple outpatient management. Acta Orthop Belg 2005; 71: 528-34.

21.Stapczynski JS. Localized depigmentation after steroid injection of a ganglion cyst on the hand. Ann Emerg Med 1991; 20: 807-9.

22.Zhang S, Xu B, Lao Y, Lu D. The Treatment of wrist ganglion cyst by the Chinese acupotomy and crisscross thread. Res Sq 2021. doi: 10.21203/rs.3.rs-94895/v1. 